Check for updates

Cite this: RSC Adv., 2017, 7, 34655

\title{
A novel silver-loaded chitosan composite sponge with sustained silver release as a long-lasting antimicrobial dressing
}

\begin{abstract}
Xiaofei Huang, Xiaojiong Bao, Zhengke Wang (D) and Qiaoling Hu*
In this study, a new kind of sustained-release silver-loaded chitosan-based sponge was developed by a simple and green method as a long-lasting antimicrobial dressing. The embedded silver nanoparticles (AgNPs) were prepared by catechol-conjugated chitosan (CCS). The CCS-coated AgNPs (CCS-AgNPs) were incorporated into the chitosan (CS) matrix through interactions between the catechol and amino groups of CS. Well-dispersed and unaggregated CCS-AgNPs were observed in the composite sponges. Due to an interconnected multi-porous structure, the composite sponges exhibited excellent flexibility and water absorption capability, which is beneficial to remove excess exudates effectively. In silver release tests, CCS-AgNPs/CS showed sustained release, whereas the control group without catechol exhibited burst release. As a bridge to bind AgNPs into the CS matrix, catechol extended the silver release time from 1 day to at least 4 days. The prolonged silver release endows CCS-AgNPs/CS sponges with long-lasting bacteriostatic effects against both Gram positive and negative bacteria. Bacterial growth was completely inhibited for up to 3 days. Meanwhile, the embedded CCS-AgNPs significantly improved the bactericidal effect. More than $99.99 \%$ bacteria could be killed by the composite sponges, which completely satisfies antibacterial requirements for wound healing. As a double-edged sword, an excessively high silver content induced cytotoxicity in MC3T3 cells. In general, the CCS-AgNPs/CS sponge with appropriate silver content $(0.63 \mathrm{wt} \%)$ is considered as a potential candidate for wound healing dressings due to its long-lasting bacteriostatic effect, powerful bactericidal activity, and excellent

biocompatibility.
\end{abstract}

Received 8th June 2017

Accepted 3rd July 2017

DOI: $10.1039 / c 7 r a 06430 f$

rsc.li/rsc-advances

\section{Introduction}

As the largest organ in the body of vertebrates, skin plays an important role in homeostasis and prevention of invasion by microorganisms. ${ }^{1}$ To date, substantial efforts have been made to provide effective treatment for burns, abrasions, exposure to chemical/biological agents, and other skin lesions. ${ }^{2,3}$ An ideal wound dressing should maintain a moist environment on a wound interface, prevent bacterial infection and create an optimal environment to promote healing. ${ }^{4}$ Two main challenges exist in designing such an ideal dressing: excess exudates and bacterial infections. ${ }^{5}$

Because of low swelling ratio, traditional dressings for clinical application, such as cellulose gauzes and bandages, can not remove excessive exudates effectively. ${ }^{6,7}$ Many studies have verified such sponge-like dressings can promote wound healing by soaking up a mount of wound exudates (more than several times their dry weight), due to their multi-porous structure. ${ }^{\mathbf{8 , 9}}$ Among them, chitosan-based sponges are considered as

MOE Key Laboratory of Macromolecular Synthesis and Functionalization, Department of Polymer Science and Engineering, Zhejiang University, Hangzhou 310027, China. E-mail:wangzk@zju.edu.cn; huql@zju.edu.cn a potential candidate for ideal wound dressings because of their excellent biocompatible, biodegradable, antibacterial and hemostatic properties. ${ }^{\mathbf{1 0 - 1 3}}$ However, many studies have reported that pure chitosan (CS) sponge was not effective enough in killing bacteria. $^{\mathbf{1 0 , 1 2}}$ To prevent bacterial infections on a wound surface, it is very desirable to incorporate antimicrobial agents into CS sponges.

Nowadays, emergence of bacterial resistance to antimicrobial drugs has become a serious problem due to abuse of commonly available antibiotics. ${ }^{14,15}$ There is an urgent need to develop new antibacterial agents instead of antibiotics. Silver nanoparticles (AgNPs) are considered as potential antibacterial agents owing to the broad-spectrum antimicrobial activity. ${ }^{16-18} \mathrm{~A}$ number of AgNPs impregnated biomaterials have been investigated, and several commercial products are currently available. ${ }^{19,20}$ But the potential toxic effects of AgNPs towards the human body still restricts the development of AgNPs-loading CS dressing. ${ }^{21,22}$

In our previous work, we have prepared a new kind of AgNPs by catechol-conjugated chitosan (CCS) that acted as both a reducing and stabilizing agent. ${ }^{23}$ The CSS-coated AgNPs (CCSAgNPs) exhibited low cytotoxicity and effective antibacterial activities due to the biocompatible polymer coating. However, 
given the solution-state, it is inconvenient to directly apply the CCS-AgNPs colloid in clinical treatments, especially in prehospital emergency care. Apparently, it's a win-win strategy to load CCS-AgNPs into CS sponge as a new kind wound dressing.

However, a crucial problem still remains unsolved: the burst release of silver from dressings. ${ }^{\mathbf{9} 24}$ Since diverse AgNPs-loading CS-based dressings have been reported recently, most of them loaded AgNPs just by the physical adsorption of dressings. ${ }^{19,25,26}$ For example, Wang et al. prepared a silver-congaing chitosan/ PEO dressing, and the result of silver release showed an initial relatively fast release in the first several hours. ${ }^{27}$ The too fast release rate means wound dressings have to be replaced frequently. There is a great demand to develop a AgNPs-loaded sponge with sustained release as a long-lasting antimicrobial dressing. ${ }^{4}$ To retard the release rate, AgNPs should be immobilized on dressings through interactions, such as electrostatic force, covalent bond $e t c .{ }^{24}$ For example, Zhong et al. fabricated a long-term antibacterial multilayer coatings containing AgNPs via a layer-by-layer (LBL) self-assembly technique. ${ }^{28}$ The loading of AgNPs was driven by electrostatic force. The sustained release of silver can kill planktonic and adherent bacteria to $100 \%$ during the first 4 days. However, LBL self-assembly technique is time consuming, laborious and difficult to be industrialized. It is still a challenge to develop a more simple and efficient method to prepare a sustained-release AgNPs-loaded dressing.

In this study, we aim to solve the above problem through catechol, a bifunctional group of CCS. Our previous work confirmed that AgNPs could be prepared by the reductive catechol groups of CCS. ${ }^{23}$ Meanwhile, several studies reported catechol could react with adjacent amino groups in CS backbones through Michael addition and Schiff base formation at pH 8.5. ${ }^{29,30}$ Hence, we suspect the interactions between catechol of CCS-AgNPs and amino groups of CS sponge can effectively retard the silver release process. To our best knowledge, it is the first time to utilize catechol as a bifunctional group to prepare AgNPs and bind the resulting AgNPs into CS sponge at same time. Because catechol is originally existed in CCS, there is no need to add other chemicals. The schematic diagram is shown in Fig. 1. Compared with other reported work, the preparation procedure of this work is obviously more simple and green, which shows great potential to be industrialized and applied in biological areas. ${ }^{28}$

In this study, a CCS-AgNPs/CS composite sponge was prepared by loading CCS-AgNPs into CS matrix through interaction between catechol and CS. XPS, XRD, EDS, and AAS were used to analyze the element in sponges and confirm the loading of CCS-AgNPs to CS sponge. The morphology of the composite sponges was measured by SEM, and water absorption and retention capacities were evaluated in PBS. A series of tests were utilized to evaluate silver release of the composite sponges. Bacteriostatic, bactericidal, and cytotoxic effects of CCS-AgNPs/ CS composite sponges were measured in vitro.

\section{Experimental}

\section{Materials}

Chitosan $\left(M_{\eta} 30 \mathrm{kDa}\right.$, deacetylated degree $\left.80 \%\right)$ was purchased from Zhejiang Gold Shell Pharmaceutical Co. Ltd. 3,4-dihydroxy-

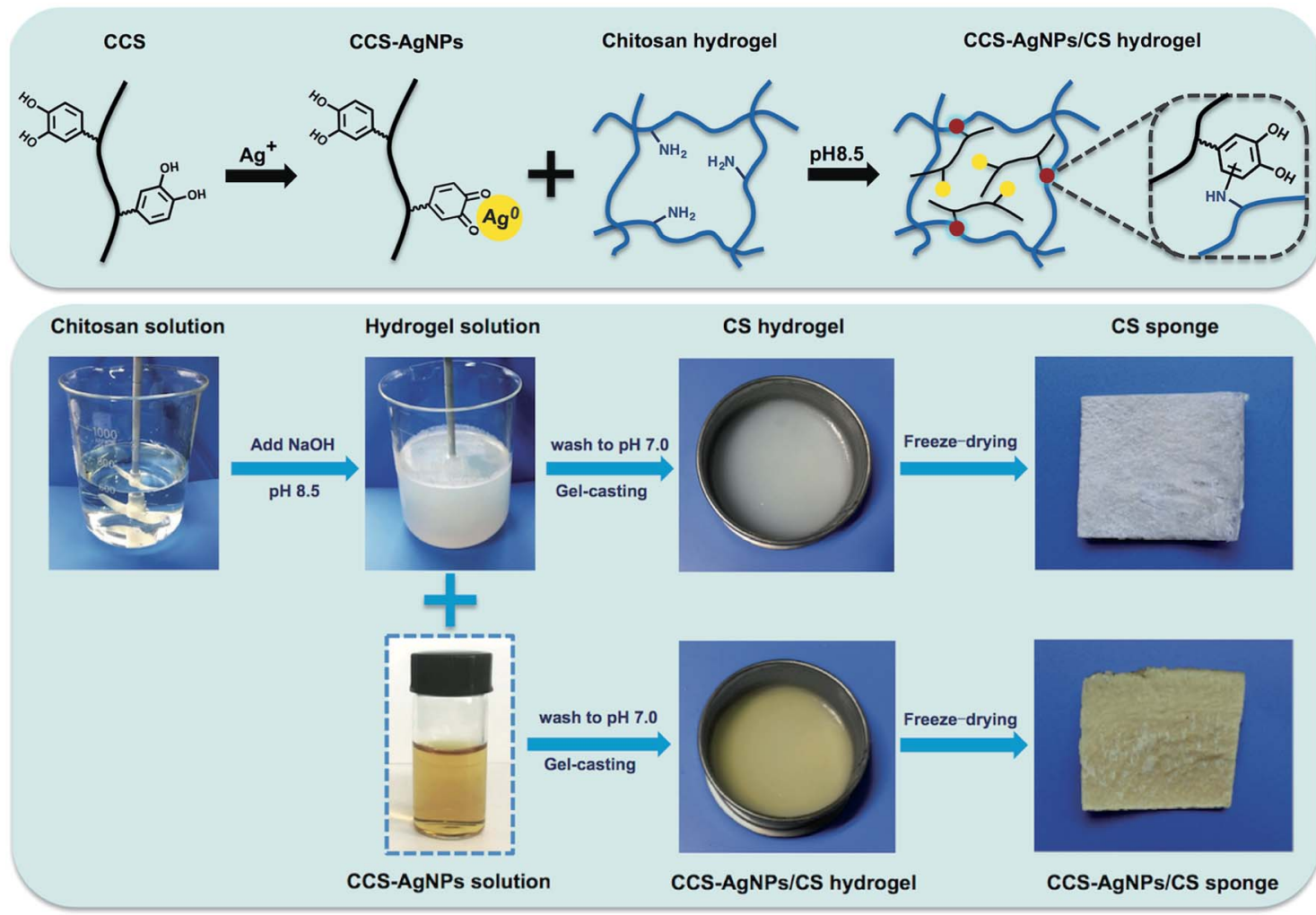

Fig. 1 Schematic representation and preparation procedure for CCS-AgNPs/CS sponges. 
hydrocinnamic acid and 1-ethyl-3-(3-dimethylaminopropyl)carbodiimide hydrochloride (EDC) were purchased from SigmaAldrich. Silver nitrate was purchased from Acros Organics. All other reagents were of analytical grade. All the aqueous solutions were prepared with distilled and deionized water (DDW).

\section{Synthesis of CCS-AgNPs/CS sponge}

According to our previous reported work, catechol-conjugated chitosan was synthesized by a standard EDC procedure and CCS-Ag NPs were prepared by a green method. ${ }^{23}$ The preparation procedure for CCS-AgNPs/CS sponges and schematic formation of the composite sponge are shown in Fig. 1. CS (3 g) was dissolved in $100 \mathrm{~mL}$ acetic acid solution $(1 \%, \mathrm{v} / \mathrm{v})$, and then $5 \mathrm{~mL} \mathrm{NaOH}$ solution $\left(0.2 \mathrm{~g} \mathrm{~mL}^{-1}\right)$ was added to obtain a homogeneous gel suspension. On one hand, the CS gel was washed to neutral by DDW and the CS sponge as control group was obtained by freeze-drying the gel. On the other hand, the CS gel was mixed with CCS-AgNPs at $\mathrm{pH} 8.5$ for $6 \mathrm{~h}$ to make catechol of CCS react with amino groups of CS gel. Then, the CCSAgNPs/CS gel was washed to neutral by DDW and freeze-dried to form the sponge. The composite sponges were coded as CS-Ag1, CS-Ag-2, and CS-Ag-3 according to various concentrations of CCS-AgNPs (100, 300, $500 \mathrm{mM})$, respectively.

\section{Chemical analysis of CCS-AgNPs/CS sponge}

The elemental composition of the composite sponges was verified using X-ray photoelectron spectroscopy (XPS, AXIS-NOVA, Kratos, Inc.) with an $\mathrm{Al} \mathrm{K} \alpha$ irradiation source. The phase structure of the composite sponges was analyzed by X-ray diffraction (XRD, Rigaku $\mathrm{D} /$ max-2550 PC, Japan) in the range of 10-80 . The distribution of AgNPs was detected by an Energy Dispersive X-ray Detector (EDX). Thermogravimetric analysis (TGA) was carried out using a Pyris Diamond TG/DTA thermal analyzer. The experiment was performed in the range of $50-600{ }^{\circ} \mathrm{C}$ at a heating rate of $10{ }^{\circ} \mathrm{C} \mathrm{min}-1$ under nitrogen atmosphere. The amount of silver of CCS-AgNPs/ CS sponges was analyzed via Atomic Absorption Spectroscopy (AAS, Hitachi 180-50, Japan). The morphology of sponges was measured by scanning electron microscopy (SEM).

\section{Mechanical properties}

The mechanical properties of the composite sponges were measured using a universal material testing instrument. The samples (measuring $10 \times 10 \times 5 \mathrm{~mm}$ ) were prepared and subjected to tensile tests at a constant rate of $30 \mathrm{~mm} \mathrm{~min}^{-1}$. The same sample was tested repeatedly five times.

\section{Water absorption and retention capacities test}

The water absorption capacity $\left(W_{\mathrm{a}}\right)$ of the samples was measured using a modified British Pharmacopoeia method. ${ }^{9,31}$ First, a circular sample, $2 \mathrm{~cm}$ in diameter, was weighed $\left(W_{\mathrm{i}}\right)$ and then immersed in $50 \mathrm{~mL}$ of $0.1 \mathrm{M}$ phosphate buffered saline (PBS) solution at $37^{\circ} \mathrm{C}$ for $1 \mathrm{~h}$. Then the sample was weighed again $\left(W_{\mathrm{j}}\right)$ after removing the surface water of samples by filter paper. $W_{\mathrm{a}}(\%)$ was calculated as $\left(W_{\mathrm{j}}-W_{\mathrm{i}}\right) / W_{\mathrm{i}} \times 100 \%$.
The water retention ability $\left(W_{\mathrm{r}}\right)$ of the samples was tested according to previous work. ${ }^{5}$ Briefly, the fully PBS-absorbed sample was placed in a desiccator containing silica gel at $37^{\circ} \mathrm{C}$ for $24 \mathrm{~h}$. The weights of fully PBS-absorbed samples $\left(W_{\mathrm{m}}\right)$, samples removed from the desiccator $\left(W_{\mathrm{n}}\right)$, and dry samples $\left(W_{\mathrm{d}}\right)$ were recorded. The $W_{\mathrm{r}}(\%)$ was calculated as $\left(W_{\mathrm{m}}-W_{\mathrm{n}}\right) / W_{\mathrm{d}}$ $\times 100 \%$. All of the tests were performed in triplicate for each independent experiment.

\section{Silver release test}

The amount of silver released from the composite sponges was analyzed via AAS. ${ }^{3}$ The samples were cut into $1 \mathrm{~cm} \times 1.5 \mathrm{~cm}$ pieces. After weighted, the specimens were placed into centrifugation tubes with $10 \mathrm{~mL}$ of PBS and were then incubated for $72 \mathrm{~h}$ at $37^{\circ} \mathrm{C} .2 \mathrm{~mL}$ of each released solution was extracted at a series of time intervals and analyzed via AAS, and then added back to $2 \mathrm{~mL}$ of fresh PBS to keep the total amount. Finally, the sample was dissolved by acetic acid solution to evaluate the total silver amount. All of the tests were performed in triplicate.

\section{Bacterial culture}

E. coli and $S$. aureus were used as model microorganisms. The bacterial strains were cultured in Luria Bertani (LB) broth and incubated in a shaking incubator at $37{ }^{\circ} \mathrm{C}$ overnight. The bacterial cells were brought into log phase by reinoculating the overnight culture $1: 100$ into LB broth and growing at $37^{\circ} \mathrm{C}$ in the shaking incubator for several hours till an optical density at $600 \mathrm{~nm}\left(\mathrm{OD}_{600}\right)$ of 0.1 was reached. ${ }^{32}$

\section{Disk diffusion test}

The disk diffusion test was a kind of leaching antimicrobial tests for evaluating the antibacterial activities. Disc shaped samples of equal weight with $5 \mathrm{~mm}$ diameter were used for the study. Bacterial suspensions were separately added to tryptic soy agar media prior to plating and then samples were placed on it. Agar plates containing samples were incubated overnight at $37{ }^{\circ} \mathrm{C}$ and the zones of inhibition surrounding the sample were measured. The samples were then gently washed thrice with $200 \mu \mathrm{L}$ of DDW. The attached microbial cells were stained with $50 \mu \mathrm{L}$ of $0.1 \%$ crystal violet dye for $15 \mathrm{~min}$. After rinsing twice with $200 \mu \mathrm{L}$ of DDW, the samples were taken photos.

\section{Bacterial suspension assays}

Bacterial suspension assay was used to investigate the bacteriostatic action of samples. Bacteria suspension was used as the starting bacterial solution (control), and sponges with and without silver nanoparticles were used as test samples. The samples $(50 \mathrm{mg})$ were respectively placed into centrifugation tubes, and $10 \mathrm{~mL}$ of nutrient broth with bacterial suspension $\left(\mathrm{OD}_{600}, 0.1\right)$ was added. The tubes were placed in a shaker incubator for $72 \mathrm{~h}$ and $\mathrm{OD}_{600}$ was noted at different time points. The assay was performed in triplicate.

To further assess the bactericidal action, a viable cell count method was used to quantitatively evaluate the bacterial reduction before and after exposed to sponges. The bacterial 
suspension was resuspended in PBS $\left(10^{5} \mathrm{CFU} \mathrm{mL}^{-1}\right)$. The samples were immersed in bacterial suspension and incubated at $37{ }^{\circ} \mathrm{C}$ for $24 \mathrm{~h}$. The number of viable bacterial cells was analyzed using the colony forming units counting. $V_{\mathrm{c}}$ and $V_{\mathrm{s}}$ are the number of viable cells before and after shake. The bacterial reduction (\%) of samples was calculated as $\left(V_{\mathrm{c}}-V_{\mathrm{s}}\right) / V_{\mathrm{c}} \times 100 \%$. All of the tests were performed in triplicate.

\section{Cell cytotoxicity assay}

Cytotoxicity of sponges to mammalian cells was measured in vitro by a CCK (Dojindo's cell counting kit-8) assay. Neonatal mouse calvaria-derived MC3T3-E1 osteoblasts were obtained from Cell Bank of Shanghai Institute of Biochemistry and Cell Biology, Chinese Academy of Sciences (Shanghai, China). Cell suspension of $1 \mathrm{~mL}$ was seeded into a 24-well plate at a density of $1 \times 10^{4}$ cells per well. Samples of the same weight $(50 \mathrm{mg})$ were immersed in cell suspension and incubated at $37{ }^{\circ} \mathrm{C}$ for $24 \mathrm{~h}, 48 \mathrm{~h}$ and $72 \mathrm{~h}$, respectively. Then $200 \mu \mathrm{L}$ of cultured medium was transferred to a 96-well plate, and $20 \mu \mathrm{L}$ of a CCK-8 solution was added to each well and incubated for $1.5 \mathrm{~h}$. Cell proliferation results are presented as optical density (OD) measured at $450 \mathrm{~nm}$ using a microplate reader (Sunrise Tecan, Austria). Three independent experiments were performed for each assay condition.

\section{Statistical analysis}

Statistical analysis was performed using one-way analysis of variance (ANOVA) followed by the Tukey's post hoc test using Origin Software (OriginLab 7.0). The probability value $(P)<0.05$ was considered to indicate significant difference.

\section{Results and discussion}

\section{Chemical analysis of CCS-AgNPs/CS sponge}

As shown in Fig. 1, a new kind of silver-loaded CS sponge was successfully prepared by loading of CCS-AgNPs at $\mathrm{pH}$ 8.5. The as-prepared sponge shows yellow due to the embedded AgNPs. A series of measurements, including XPS, XRD, EDS and AAS, were used to analyze the element in sponges and confirm the loading of CCS-AgNPs to CS sponges.
Fig. 2a shows the elemental composition of the composite sponge (CS-Ag-1) verified by XPS. Two peaks at $374 \mathrm{eV}$ and $368 \mathrm{eV}$ corresponded with $\mathrm{Ag} 3 \mathrm{~d}_{3 / 2}$ and $\mathrm{Ag} 3 \mathrm{~d}_{5 / 2}$ binding energies, consistent with the reported data of metallic Ag. ${ }^{33}$ The XPS results confirmed the existence of $\mathrm{Ag}$ on the composite sponge.

The XRD patterns (Fig. 2b) further confirmed embedded AgNPs in CCS-AgNPs/CS sponges. Compared with CS sponges, a new peak at 38.1 which is assigned to the (111) of metallic Ag, was observed in all of CCS-AgNPs/CS sponges. The intensity of the peak depends on $\mathrm{Ag}$ content. It is obvious that the $\mathrm{Ag}$ content of CS-Ag-3 is higher than others, but the specific content should be determined by other quantitative analysis, such as AAS.

The results of AAS shows that weight percentage (wt $\%$ )of $\mathrm{Ag}$ in composite sponges from CS-Ag- 1 to CS-Ag-3 are $0.35 \pm 0.07 \%$, $0.63 \pm 0.09 \%$, and $1.42 \pm 0.25 \%$, respectively.

The morphology and distribution of the AgNPs on the composite sponge surface were assessed by SEM and EDS. A number of spherical nanoparticles with good dispersion were observed in the SEM photos (Fig. 2c, insert). The corresponding EDS spectra confirmed that the composition of the particles was silver. As we know, the dispersity of silver nanoparticles is considered to be the most important factor with respect to their antibacterial efficacy: the better the dispersibility, the higher the antibacterial activity. ${ }^{34}$ Therefore, the embedded AgNPs with good dispersion in the composite sponge may endow the sponge with remarkable antibacterial activity.

\section{Morphology of sponges}

Compared with fibers and membranes, sponges could absorb more exudates to promote wound healing, due to their micropore structure. ${ }^{35}$ Therefore, the porous morphology is a key property of sponges, which could be evaluated by SEM. The inner morphologies of CS and CCS-AgNPs/CS sponges are shown in Fig. 3. Compared with the pure CS sponge, the porous structure of the composite sponge does not change remarkably after loading of CCS-AgNPs. All of sponges, which were prepared via lyophilization of hydrogel, exhibited high porosity and wellinterconnected micropore structure.
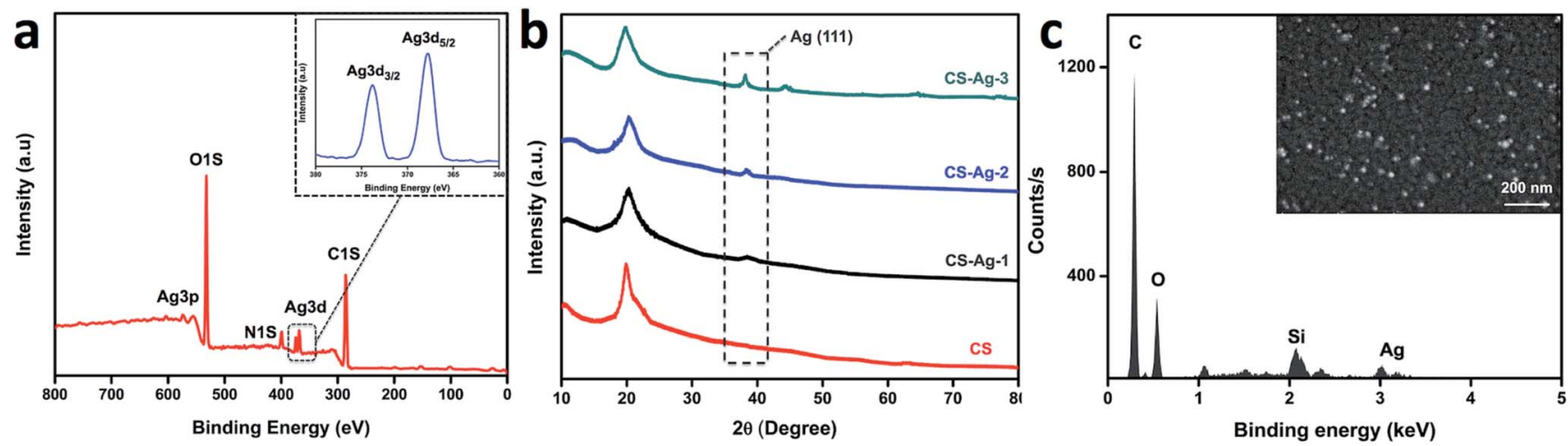

Fig. 2 (a) XPS fully scanned spectra of CS-Ag-2 and XPS spectra of Ag 3d; (b) XRD patterns of CS, CS-Ag-1, CS-Ag-2, and CS-Ag-3; (c) insert shows a SEM image of CS-Ag-2 sponge and EDS spectra corresponded to the SEM image. 

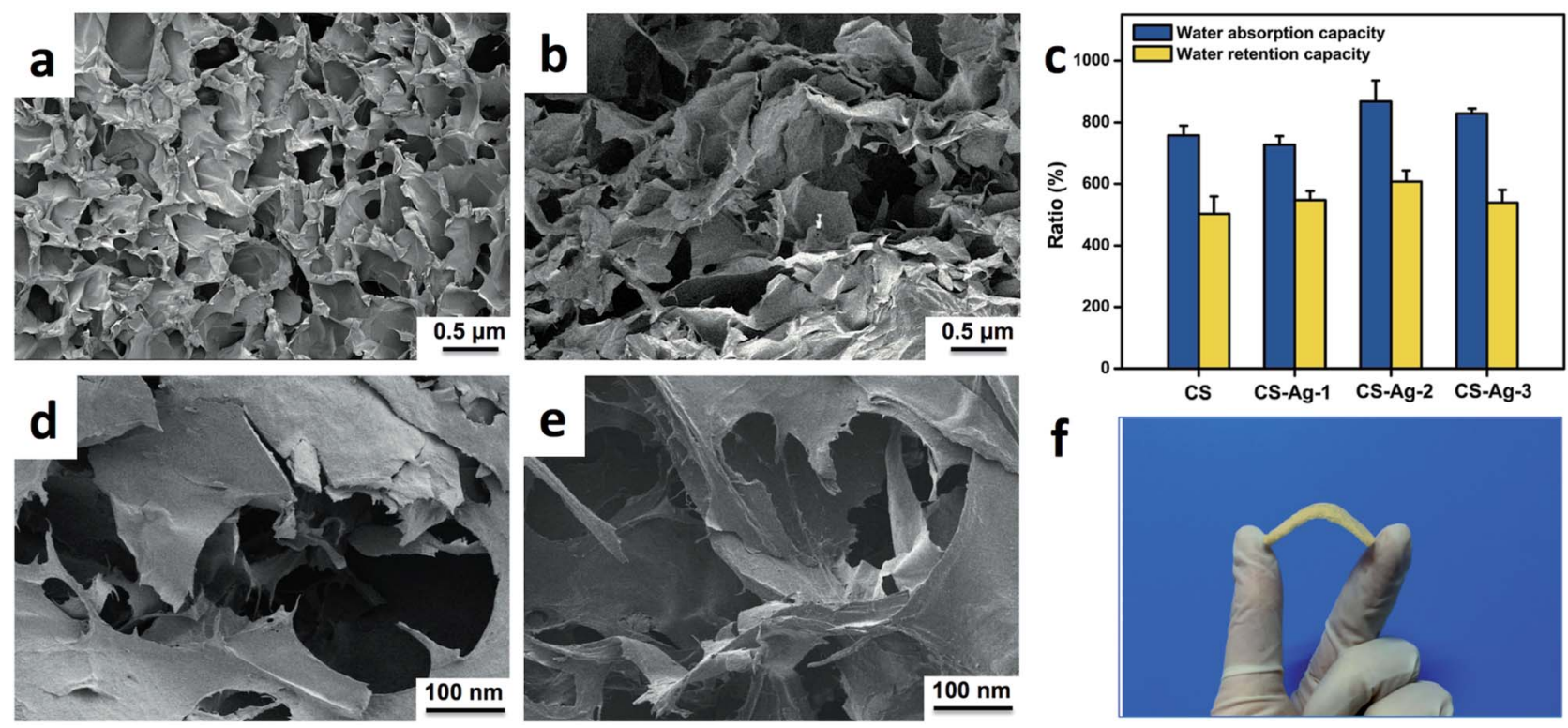

Fig. 3 SEM images of sponges: (a, d) CS sponge; (b, e) CS-Ag-2 sponge; (c) the water absorption and retention capabilities of CS, CS-Ag-1, CS$\mathrm{Ag}-2$, and $\mathrm{CS}-\mathrm{Ag}-3$; ( $\mathrm{f}$ ) photo shows the flexibility of CS-Ag-2 sponge.

Due to the 3-D interconnected pores, the CCS-AgNPs/CS sponge exhibits excellent flexibility in Fig. 3f. Table 1 shows the mechanical test results of CS sponge and CCS-AgNPs/CS sponges. Compared with CS sponge, CCS-AgNPs/CS sponges exhibit similar mechanical test results $(p>0.05)$, which indicates the embedded AgNPs wouldn't influence the mechanical properties of sponges. As shown in Table 1, the tensile strength of CCS-AgNPs/CS sponges is in the range of $0.2-0.3 \mathrm{MPa}$, and the elongation at break is in the range of $13-17 \%$. According to the published literature, the mechanical properties of CCSAgNPs/CS sponge meet the requirements of clinic applications. ${ }^{36,37}$

\section{Water absorption and retention capabilities}

As mentioned above, the micro-porous architecture of CCSAgNPs/CS sponges may endow the materials with a good water absorption capacity. Fig. 3c shows the results of water absorption and retention capabilities of sponges. The CS sponge could absorb more than 12 times its dry weight in PBS. Meanwhile, the CS sponge could still retain a mount of PBS more than 8 times its dry weight when exposed to air over $24 \mathrm{~h}$.

Table 1 Mechanical test results of CS sponge and CCS-AgNPs/CS sponges $^{a}$

\begin{tabular}{lll}
\hline Samples & Tensile strength $(\mathrm{MPa})$ & Elongation at break $(\%)$ \\
\hline CS & $0.28 \pm 0.04$ & $14.70 \pm 0.92$ \\
CS-Ag-1 & $0.27 \pm 0.03^{\Omega}$ & $15.04 \pm 1.05 \Omega$ \\
CS-Ag-2 & $0.31 \pm 0.05$ & $14.29 \pm 0.88^{\Omega}$ \\
CS-Ag-3 & $0.24 \pm 0.04 \Omega$ & $16.22 \pm 1.65 \Omega$
\end{tabular}

${ }^{a}$ Note: data presented as mean $\pm \mathrm{SD}, n=3$. $\Omega$ : non-significant difference compare to CS sponge $(p>0.05)$.
The excellent capability of water retention is beneficial to maintain a moist environment on the wound interface. Compared with pure CS sponges, the composite sponges exhibited similar water absorption and retention capabilities ( $p>0.05)$, which is consistent with the SEM result. The result indicates the embedded AgNPs would not affect the physical properties of sponges, such as porosity and swelling ratio. Compared with other reported work, the CCS-AgNPs/CS sponges exhibit more excellent water absorption and retention capabilities, which indicates the potential as an ideal wound dressing. ${ }^{38}$

\section{Silver release of CCS-AgNPs/CS sponge}

The main aim of this work is to design a new silver-loaded CS sponge as a long-lasting antimicrobial dressing. Through interactions between catechol and amino groups of CS, we hope to bind the CCS-AgNPs into CS sponges to retard the silver release process (Fig. 1). To confirm the function of catechol, we prepared a new kind of silver-loaded CS sponge as a control (name as CS-AgNPs/CS) by replacing CCS with CS. The only difference between the control group (CS-AgNPs/CS) and experimental group (CCS-AgNPs/CS) is the absence of catechol. Fig. 4 shows the release rate of silver from the composite sponges in PBS during $96 \mathrm{~h}$. Within the first day, a mount of Ag was quickly released from the control group, and then only a little of silver release was observed over the following 3 days. No significant difference of release amount is observed among $72 \mathrm{~h}$ and $96 \mathrm{~h}(p>0.05)$. In contrast, all of experimental groups (CS-Ag-1, CS-Ag-2 and CS-Ag-3) show sustained release during 4 days. There is a significant difference between silver release amount at $72 \mathrm{~h}$ and $96 \mathrm{~h}(p<0.01)$. The results confirmed our suspicion that catechol could retard silver release rate by reacting with adjacent amino groups in CS backbones through Michael addition and Schiff base formation. As reported in 


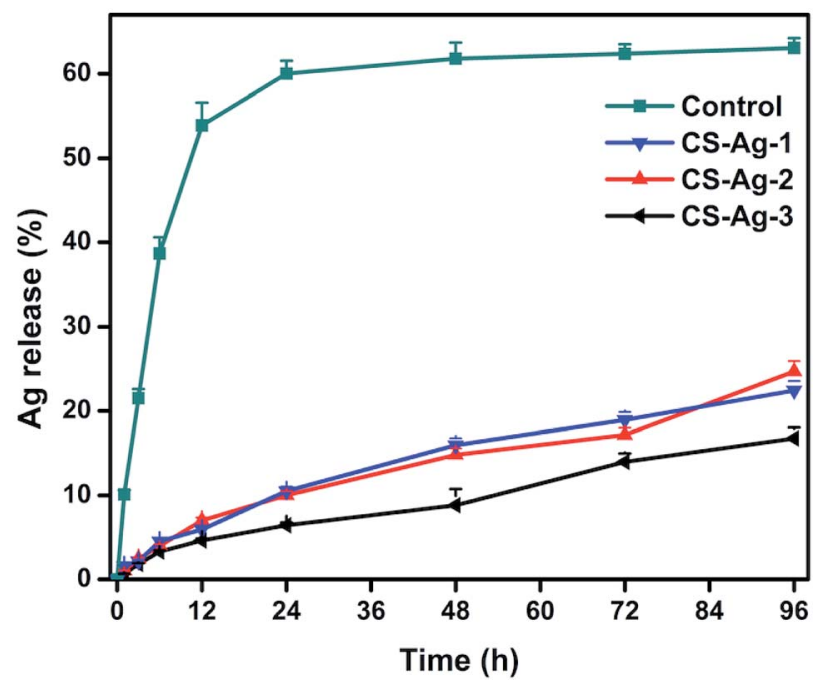

Fig. 4 Silver release of CS-Ag-1, CS-Ag-2, and CS-Ag-3; CS-AgNPs/ CS sponge is set as control.

other papers, similar results confirmed catechol is a potential bridge to bind nanoparticles into polymer matrix due to excellent chemical reactivity. ${ }^{39} \mathrm{Compared}$ to Wang et al. research, the silver release time of CCS-AgNPs/CS sponge is nearly 4 times than other existing silver-loaded dressing. ${ }^{27}$

To further confirm sustained silver release, we used a kind of leaching antimicrobial test (disk diffusion test) to evaluate the CS-based sponges. Fig. $5 \mathrm{a}$ and $\mathrm{b}$ show the results of the disk diffusion test for E. coli and S. aureus, respectively. The zone of inhibition depends on the release of antimicrobial component. ${ }^{25}$ For the pure CS sponge, there is nothing to release from the water-insoluble sponge. As expected, there is no inhibition zone observed on the CS sponge. A CCS-AgNPs solution (named as Ag-2) with same silver amount was used as a control to compare with the CS-Ag-2 sponge. According to the test principle, the more the amount of silver release, and the bigger the zone of inhibition. The results show the inhibition zones around the CS-Ag-2 sponge are much smaller than the CCSAgNPs solution, indicating that the CS-Ag-2 sponge only released a part of silver during the test $(24 \mathrm{~h})$. As shown in the release profile of silver (Fig. 4), the CS-Ag-2 sponge only released $10.6 \%$ silver within $24 \mathrm{~h}$. Therefore the disc diffusion test further confirmed the sustained silver release of CCS-AgNPs/CS sponges.

As mentioned above, about $89.4 \%$ of silver remained in the CS-Ag-2 sponge after incubation for 1 day, which may have an remarkable bacteriostatic effect through direct contact with bacteria. To confirm our suspicion, we used crystal violet to stain attached microbial cells in sponges after disc diffusion tests. ${ }^{40}$ The CS sponge untreated with bacteria (named as CS-) was taken as negative control, while the CS sponge treated with bacteria (named as $\mathrm{CS}+$ ) was taken as positive control. As shown in Fig. 5c, the CS- sponge was not stained by crystal violet, because no bacteria existed in the sponge. The whole CS+ sponge was stained purple by crystal violet, indicating that a pure CS sponge could not resist the growth of bacteria. For the CS-Ag-2 sponge, only the surface contacting with bacteria was stained purple and the rest of the sponge remained uncolored. The result confirmed our suspicion that CCS-AgNPs/CS sponges could significantly inhibit the growth of contacted bacteria.

\section{Bacteriostatic action of CCS-AgNPs/CS sponges}

To further quantitatively evaluate the bacteriostatic action of CCS-AgNPs/CS sponges, we used bacterial suspension assay to analyze the bacterial growth. Fig. 6 shows the growth curves of E. coli and $S$. aureus after incubation in LB broth with CS and CCS-AgNPs/CS sponges during $72 \mathrm{~h}$, respectively. As a negative control, the untreated $E$. coli and $S$. aureus bacteria showed typical three phases of growth (lag, exponential and stabilization phase). The untreated bacterial cells had promptly reached the exponential phase, but when bacteria were exposed to CS sponges, their lag phase was delayed approximately $4 \mathrm{~h}$ and $2 \mathrm{~h}$ for $E$. coli and $S$. aureus, respectively. It revealed the CS sponge could tightly inhibit the bacterial growth due to the antibacterial effect of CS. There is no exponential phase of growth observed after $12 \mathrm{~h}$ incubated with CCS-AgNPs/CS sponges. To figure out the difference among the three kinds of composite sponges, the incubation time lasted up to 3 days. Fig. $6 \mathrm{~b}$ and d show the exponential phase was delayed $24 \mathrm{~h}$ by CS-Ag- 1 , while CS-Ag-2 and CS-Ag-3 could completely inhibit bacterial growth for up to 3 days. The difference was induced by the
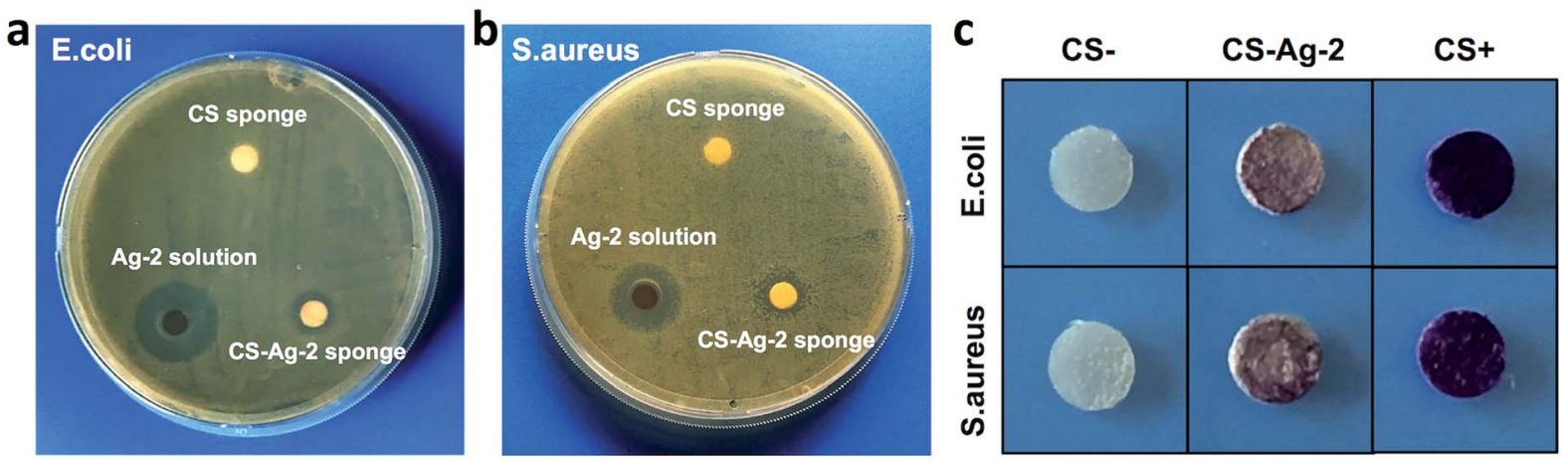

Fig. 5 Inhibition zones of CS sponge, CS-Ag-2 sponge and Ag-2 solution against E. coli (a) and S. aureus (b), Ag-2 is a CCS-AgNPs solution containing the same silver amount as CS-Ag-2 sponge; (c) photos of samples stained by crystal violet after disc diffusion test. 

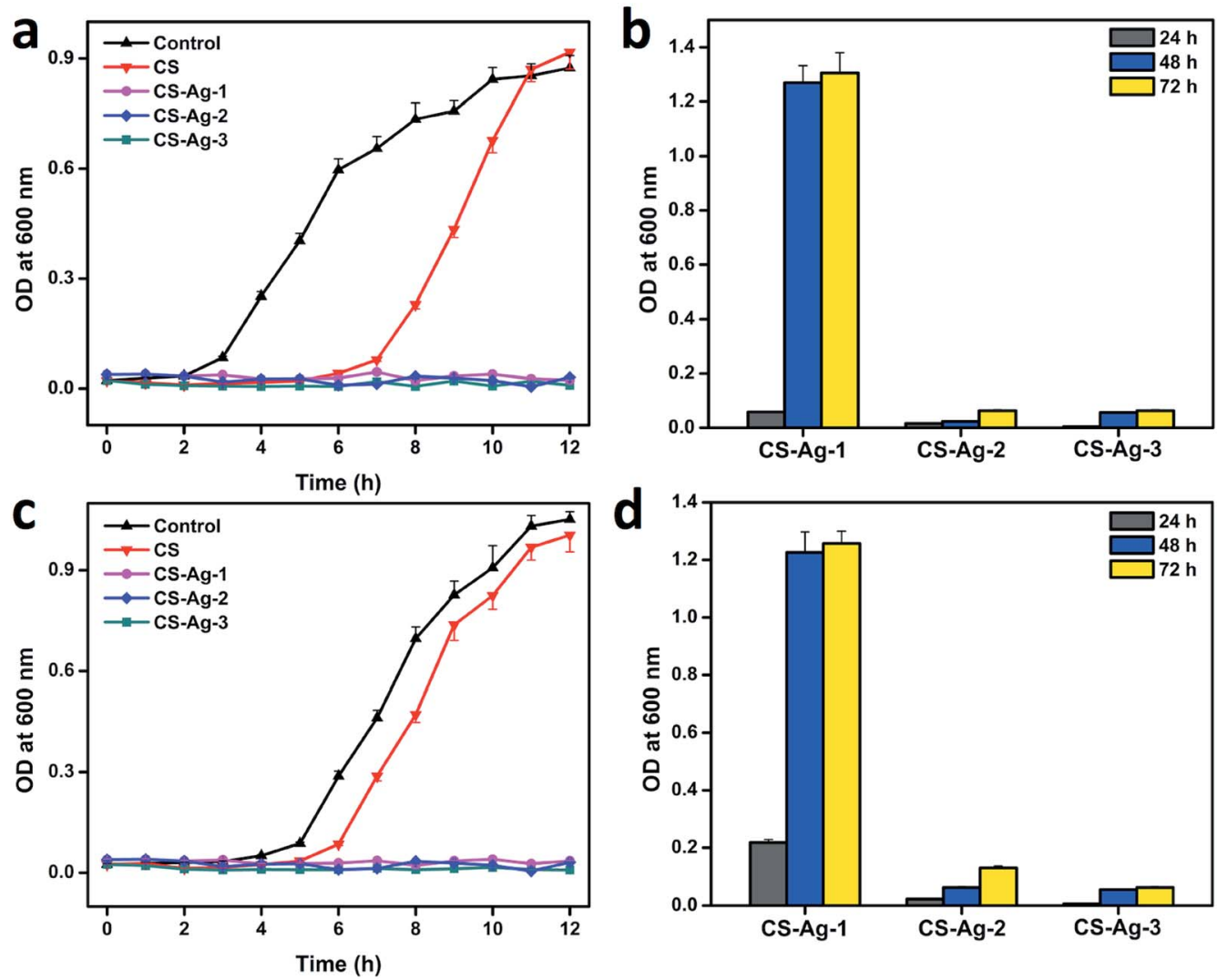

Fig. 6 Bacterial growth curve of E. coli ( $a$ and b) and S. aureus (c and d) after exposed to CS, CS-Ag-1, CS-Ag-2, and CS-Ag-3 sponges.

amount of silver-loaded in the composite sponges. Compared with other antibacterial materials, the long-last bacteriostatic action of CCS-AgNPs/CS sponges is amazing. ${ }^{41}$

\section{Bactericidal effect of CCS-AgNPs/CS sponges}

To further assess the bacterial killing capability, a viable cell count method was used to quantitatively evaluate the bacterial reduction before and after exposed to sponges. Fig. 7 shows the

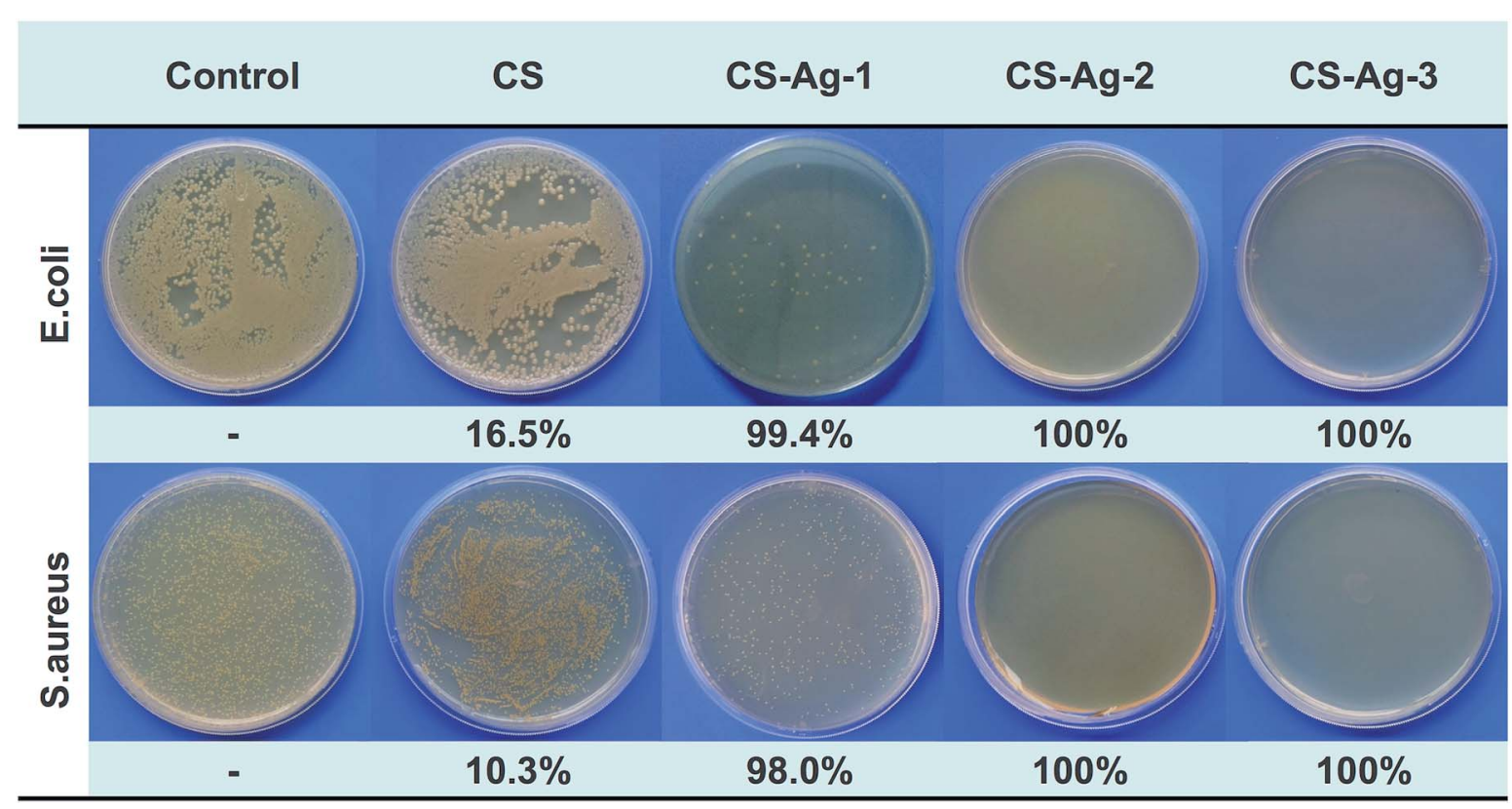

Fig. 7 Images of bacteria colony and viable cell reduction before and after exposed to CS, CS-Ag-1, CS-Ag-2, and CS-Ag-3 sponges. 


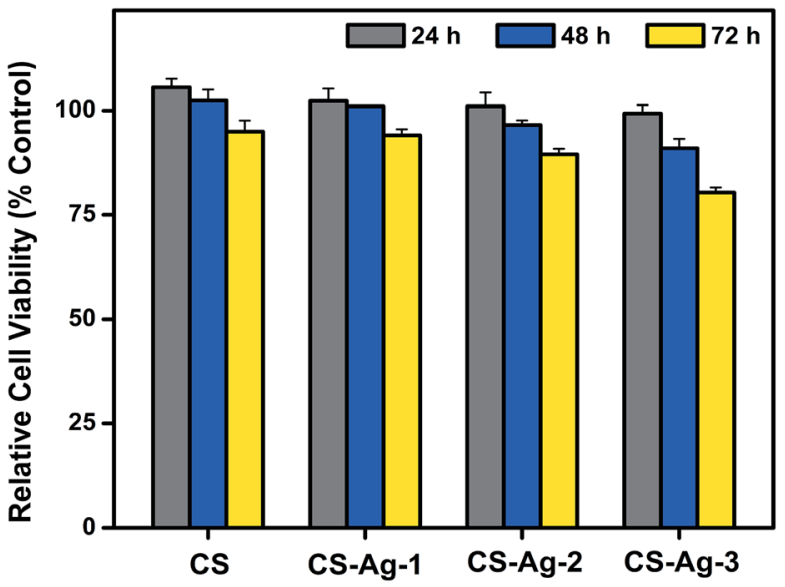

Fig. 8 Relative cell viabilities of MC3T3 cells after incubation for different times $(24,48$ and 72 h) with CS, CS-Ag-1, CS-Ag-2, and CS$\mathrm{Ag}-3$ sponges.

images of bacteria colony after exposed to CS and CCS-AgNPs/ CS sponges against E. coli and S. aureus. As expected, the CS sponge reduced a negligible bacterial reduction of less than $17 \%$, while all of CCS-AgNPs/CS sponges led to over 98\% bacterial reduction. Thanks to the loaded silver, the composite sponges exhibited remarkable bactericidal effect against both Gram-positive $S$. aureus and Gram-negative E. coli. The initial bacterial counts of $E$. coli and $S$. aureus were $5 \log \mathrm{CFU} \mathrm{ml} \mathrm{m}^{-1}$. About 4-log reduction in counts was observed in the presence of CS-Ag-2 and CS-Ag-3, indicating that more than $99.99 \%$ bacteria were killed. It is a very high kill ratio of bacteria for antibacterial materials. ${ }^{19}$ The powerfully bactericidal effect of CCS-AgNPs/CS sponges may be due to a couple of reasons. On one hand, the embedded CCS-AgNPs with good dispersion could exhibit significant antibacterial activity. On the other hand, the excellent water absorption and retention capabilities make CCSAgNPs highly contact with bacteria.

\section{Cytotoxic effect in vitro}

Despite the potent antibacterial activity, the biological applications of CCS-AgNPs/CS sponges are limited due to their potential cytotoxicity against mammalian cells. As a cell viability assay, CCK-8 assay was utilized to study the cytotoxic effect of CCS-AgNPs/CS sponges in vitro. Fig. 8 shows the relative cell viabilities of MC3T3 cells for different culture times with samples. As expected, the CS sponge exhibited excellent biocompatibility by promoting the growth of cells at the first 2 days. The relative cell viabilities were more than $90 \%$ after treatment with CS-Ag-1 and CS-Ag-2 sponges for $72 \mathrm{~h}$. For the CS-Ag-3 group, a sharp drop of cell viabilities was observed after $72 \mathrm{~h}$, which is caused by excessively high silver content. It is obvious that the silver content increased the cytotoxic effect of CCS-AgNPs/CS sponges.

\section{Conclusions}

In this study, we aim to develop a new kind of sustained-release silver-loaded chitosan-based sponge by a simple and green method as a long-lasting antimicrobial dressing. Through interactions between catechol and amino groups of CS, CCSAgNPs were bound into the CS sponge to retard the silver release process. A series of CCS-AgNPs/CS sponges with different silver contents $(0.35,0.63,1.42 \mathrm{wt} \%)$ were prepared and coded as CS-Ag-1, CS-Ag-2, and CS-Ag-3. XPS, XRD, and EDS confirmed the incorporation of CCS-AgNPs and well-dispersed and unaggregated CCS-AgNPs were observed in the composite sponges. Due to interconnected multi-porous structure, the composite sponges exhibited excellent flexibility and water absorption capability, which is beneficial to remove excessive exudates effectively.

In silver release tests, CCS-AgNPs/CS showed sustained release, whereas the control group without catechol (CS-AgNPs/ CS) exhibited burst release. The existence of catechol in CCSAgNPs/CS sponges reduced the amount of silver release by nearly $67 \%$ but extended the release time from 1 day to at least 4 days. A leaching antimicrobial test further verified the slow release of silver in CCS-AgNPs/CS sponges, which is a crucial prerequisite of a prolonged antibacterial activity.

The bacteriostatic assay exhibited the CS-Ag-2 and CS-Ag-3 sponges could completely inhibit bacterial growth for up to 3 days. Meanwhile, the bactericidal assay showed the CS-Ag-2 and CS-Ag-3 sponges could kill more than $99.99 \%$ bacteria. The CCSAgNPs/CS sponges with relatively high silver content (more than $0.63 \mathrm{wt} \%$ ) exhibited remarkable antibacterial effect against both Gram-positive $S$. aureus and Gram-negative E. coli. However, cell cytotoxicity assay revealed excessively high silver content of composite sponges would also increase the cytotoxic effect as a double-edged sword. In general, CS-Ag-2, a CCS-AgNPs/CS sponge with proper silver content, is considered as a potent candidate for wound healing dressings due to the long-lasting bacteriostatic effect, powerfully bactericidal activity, and excellent biocompatibility.

\section{Acknowledgements}

This work was financially supported by the Fundamental Research Funds for the Central Universities, and the National Natural Science Foundation of China (No. 21374099 and 51473144).

\section{References}

1 B. Moghadas, E. Dashtimoghadam, H. Mirzadeh, F. Seidi and M. M. Hasani-Sadrabadi, RSC Adv., 2016, 6, 7701-7711.

2 A. GhavamiNejad, A. Rajan Unnithan, A. Ramachandra Kurup Sasikala, M. Samarikhalaj, R. G. Thomas, Y. Y. Jeong, S. Nasseri, P. Murugesan, D. Wu, C. Hee Park and C. S. Kim, ACS Appl. Mater. Interfaces, 2015, 7, 1217612183.

3 A. GhavamiNejad, C. H. Park and C. S. Kim, Biomacromolecules, 2016, 17, 1213-1223.

4 J. J. Elsner, D. Egozi, Y. Ullmann, I. Berdicevsky, A. ShefyPeleg and M. Zilberman, Burns, 2011, 37, 896-904.

5 D. Ye, Z. Zhong, H. Xu, C. Chang, Z. Yang, Y. Wang, Q. Ye and L. Zhang, Cellulose, 2015, 23, 749-763. 
6 R. Singh and D. Singh, Int. Wound J., 2014, 11, 264-268.

7 F. L. Mi, Y. B. Wu, S. S. Shyu, A. C. Chao, J. Y. Lai and C. C. Su, J. Membr. Sci., 2003, 212, 237-254.

8 P. I. Siafaka, A. P. Zisi, M. K. Exindari, I. D. Karantas and D. N. Bikiaris, Carbohydr. Polym., 2016, 143, 90-99.

9 B. S. Anisha, R. Biswas, K. P. Chennazhi and R. Jayakumar, Int. J. Biol. Macromol., 2013, 62, 310-320.

10 H. Zhang, X. Lv, X. Zhang, H. Wang, H. Deng, Y. Li, X. Xu, R. Huang and X. Li, RSC Adv., 2015, 5, 50523-50531.

11 X. Huang, J.-w. Jia, Z.-k. Wang and Q.-l. Hu, Chin. J. Polym. Sci., 2014, 33, 284-290.

12 S.-Y. Ong, J. Wu, S. M. Moochhala, M.-H. Tan and J. Lu, Biomaterials, 2008, 29, 4323-4332.

13 X. Huang, Y. Sun, J. Nie, W. Lu, L. Yang, Z. Zhang, H. Yin, Z. Wang and Q. Hu, Int. J. Biol. Macromol., 2015, 75, 322-329.

14 C. Luo, W. Liu, B. Luo, J. Tian, W. Wen, M. Liu and C. Zhou, Carbohydr. Polym., 2017, 156, 235-243.

15 A. U. Khan and S. Qayyum, IET Nanobiotechnol., 2016, 10, 349-357.

16 Y. Cheng, F. Wang, C. Fang, J. Su and L. Yang, J. Alloys Compd., 2016, 658, 684-688.

17 E. A. Jun, K. M. Lim, K. Kim, O. N. Bae, J. Y. Noh, K. H. Chung and J. H. Chung, Nanotoxicology, 2011, 5, 157-167.

18 X. Huang, X. Bao, Y. Liu, Z. Wang and Q. Hu, Sci. Rep., 2017, 7, 28140.

19 M. Konop, T. Damps, A. Misicka and L. Rudnicka, J. Nanomater., 2016, 2016, 1-10.

20 F. Franck, K. S. Morley, W. Ben, B. L. Sharp, P. L. Arnold, S. M. Howdle, B. Roger, P. D. Brown, P. D. Winship and H. J. Reid, J. Antimicrob. Chemother., 2004, 54, 1019-1024.

21 S. Zhang, Y. Tang and B. Vlahovic, Nanoscale Res. Lett., 2016, 1-8.

22 H. Y. Son, J. H. Ryu, H. Lee and Y. S. Nam, Macromol. Mater. Eng., 2013, 298, 547-554.

23 X. Huang, Y. Pang, Y. Liu, Y. Zhou, Z. Wang and Q. Hu, RSC Adv., 2016, 6, 64357-64363.

24 G. Liu, K. Li, Q. Luo, H. Wang and Z. Zhang, J. Colloid Interface Sci., 2017, 490, 642-651.
25 X. Wang, F. Cheng, J. Gao and L. Wang, J. Biomater. Appl., 2014, 29, 1086-1095.

26 I. Kohsari, Z. Shariatinia and S. M. Pourmortazavi, Carbohydr. Polym., 2016, 140, 287-298.

27 X. Wang, F. Cheng, J. Gao and L. Wang, J. Biomater. Appl., 2015, 29, 1086-1095.

28 X. Zhong, Y. Song, P. Yang, Y. Wang, S. Jiang, X. Zhang and C. Li, PLoS One, 2016, 11, e0146957.

29 J. H. Ryu, S. Hong and H. Lee, Acta Biomater., 2015, 27, 101. 30 O. Zvarec, S. Purushotham, A. Masic, R. V. Ramanujan and A. Miserez, Langmuir, 2013, 29, 10899-10906.

31 Z. Pei, Q. Sun, X. Sun, Y. Wang and P. Zhao, Bio-Med. Mater. Eng., 2015, 26(1), S111-S118.

32 J. Tang, Q. Chen, L. Xu, S. Zhang, L. Feng, L. Cheng, H. Xu, Z. Liu and R. Peng, ACS Appl. Mater. Interfaces, 2013, 5, 38673874 .

33 H. Shi, H. Liu, S. Luan, D. Shi, S. Yan, C. Liu, R. K. Y. Li and J. Yin, Compos. Sci. Technol., 2016, 127, 28-35.

34 S. Arora, J. Jain, J. M. Rajwade and K. M. Paknikar, Toxicol. Lett., 2008, 179, 93-100.

35 R. Jayakumar, M. Prabaharan, P. T. Sudheesh Kumar, S. V. Nair and H. Tamura, Biotechnol. Adv., 2011, 29, 322-337.

36 B. Lu, T. Wang, Z. Li, F. Dai, L. Lv, F. Tang, K. Yu, J. Liu and G. Lan, Int. J. Biol. Macromol., 2016, 82, 884-891.

37 S. Gustaite, J. Kazlauske, J. Bobokalonov, S. Perni, V. Dutschk, J. Liesiene and P. Prokopovich, Colloids Surf., A, 2015, 480, 336-342.

38 D. Liang, Z. Lu, H. Yang, J. Gao and R. Chen, ACS Appl. Mater. Interfaces, 2016, 8, 3958-3968.

39 Y. Chan Choi, J. S. Choi, Y. J. Jung and Y. W. Cho, J. Mater. Chem. B, 2014, 2, 201-209.

40 V. Alt, T. Bechert, P. Steinrücke, M. Wagener, P. Seidel, E. Dingeldein, E. Domann and R. Schnettler, Biomaterials, 2004, 25, 4383-4391.

41 Y.-W. Wang, H. Tang, W. Di, D. Liu, Y. Liu, A. Cao and H. Wang, Environ. Sci.: Nano, 2016, 3, 788-798. 